\section{Main results of the international intercomparison of passive radon detectors under field conditions in Marie Curie's tunnel in Lurisia (Italy)}

\author{
Francesco Cardellini, \\ Enrico Chiaberto, Luisella Garlati, \\ Daniele Giuffrida, \\ Federica Leonardi, \\ Mauro Magnoni, \\ Gianfranco Minchillo, \\ Anna Prandstatter, Elena Serena, \\ Rosabianca Trevisi, \\ Rosamaria Tripodi, Miriam Veschetti
}

\begin{abstract}
In recent years a large number of radon intercomparison exercises has been organized; most of them took place in radon chambers, in reference atmosphere of the parameter to control (i.e. radon gas) under temperature, humidity and atmospheric pressure stable conditions. In 2014, in the tunnel belonging to the Lurisia spas complex (Lurisia, Piedmont, Italy), with natural high concentrations of uranium and radon gas, an intercomparison exercise has been held to give to radon measurement services and laboratories the possibility to test their passive systems under field conditions, which are less controlled and much more challenging. The response of laboratories was very positive: 46 participants from 10 European countries and 3 non-European countries. Generally about $80 \%$ of results of participants were considered acceptable even if it was observed a global trend of a substantial underestimation of the actual radon concentration.
\end{abstract}

Key words: in-field conditions $\bullet$ intercomparison $\bullet$ passive detectors $\bullet$ radon

F. Cardellini

ENEA/INMRI,

Rome, Italy

E. Chiaberto, M. Magnoni, A. Prandstatter, E. Serena,

R. Tripodi

Agenzia Regionale per la Protezione Ambuentale

(ARPA), Piemonte, Ivrea, Italy

L. Garlati

Politecnico di Milano, Milano, Italy

D. Giuffrida

Federal Authority for Nuclear Regulation (FANR),

United Arab Emirates

G. Minchillo

Joint Research Centre (JRC), Ispra, Italy

F. Leonardi ${ }^{\bowtie}$, R. Trevisi, M. Veschetti

Italian National Workers Compensation Authority

(INAIL),

Department of Medicine, Epidemiology, Occupational and Environmental Hygiene,

Via Fontana Candida 1, 00040 Monteporzio Catone

(Rome) Italy,

Tel.: + 390694181265 ,

E-mail: f.leonardi@inail.it

Received: 22 December 2015

Accepted: 24 March 2016

\section{Introduction}

In the recent years, the request of a more adequate traceability and reliability of radon measurements gave rise to many international intercomparison exercises; intercomparisons are a very important tool for measurement services and laboratories in order to detect potential problems. These exercises, organized by several institutes, such as Public Health England (PHE, formerly Health Protection Agency - HPA, UK) [1], Bundesamt für Strahlenschutz (BfS, Germany) [2] and NIRS (National Institute of Radiological Sciences, Japan) [3], are usually performed in radon reference chamber (STAR) [4] where the radon concentration is strictly controlled, under temperature, humidity and atmospheric pressure stable conditions. However, very soon, the growing of under in-field experiences highlights that the extremely controlled conditions typical of intercomparison exercises are too far from the actual conditions encountered in dwellings and workplaces where the dosimeters are usually exposed.

The first very interesting experience of radon in-field intercomparison was done in Spain in 2011 [5]. Following the good results obtained in this previous event, it was planned to hold a similar exercise in Italy. Therefore, the Italian Radiation Protection Association (AIRP), together with other national and international institutions, organized in 2014 the international intercomparison of passive radon detectors under field conditions' in the Marie Curie's tunnel, in Lurisia, a spa location in the 
South-West of Piedmont. Nowadays, the tunnel is a suitable place for testing measurement devices and for radiation protection experiments. In the tunnel, environmental conditions are quite severe: radon concentrations are very high (above $10000 \mathrm{~Bq} / \mathrm{m}^{3}$ ) and relative humidity is near $95 \%$.

A detailed description of logistical arrangements, metrological aspects and all results were presented in the international conference held in Lurisia in May 2015 , during which the report of the intercomparison exercise was provided to each participant. In the present paper, a synthesis of main results is given.

\section{Material and methods}

\section{Metrological aspects}

The reference radon concentrations in the tunnel were measured using six active monitors: three AlphaGUARD Professional Radon Monitor (Genitron, $\mathrm{GmbH}$ ), based on an ionization chamber, and three MR1-PLUS (TESYS, Italy), based on a scintillation cell. All the monitors were calibrated in the INMRI Laboratory at the ENEA Casaccia Research Centre. More details about metrological aspects are discussed elsewhere [6].

\section{Radiometric characterization of the Lurisia tunnel site}

The Lurisia Cave consists of a main tunnel where there are some plants for collecting spring water. One of these springs, named Garbarino, is highly radioactive, up to $25000 \mathrm{~Bq} / \mathrm{l}$ of radon in water. Twenty meters away from the entrance of the tunnel, it opens a secondary cave, where there is one of the main Garbarino's wells. This site was chosen as location for the intercomparison exposures: besides very high indoor radon levels and poor ventilation rate, the other environmental parameters (temperature and humidity) are quite stable. In this cavity, approximately $5 \times 3 \mathrm{~m}^{2}$ and an average height of $1.8 \mathrm{~m}$, eight wire shelves were placed for a good air circulation amongst radon devices during the exposures. During the weeks before the intercomparison exercise, nine radon active monitors - AlphaGUARD, MR1-PLUS and Radim 5B (Jiri Plch M.Eng-SMM) - were placed on the shelves (Fig. 1), six of which are calibrated by INMRI, the others aligned with the previous ones through proper internal intercomparisons.

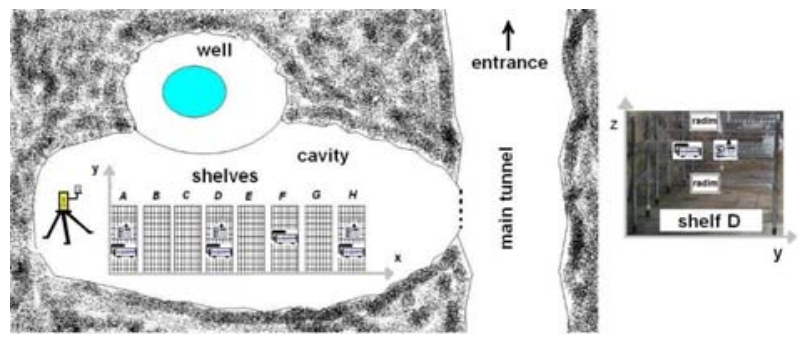

Fig. 1. Scheme of the arrangement of the monitors during the exposures. A detailed picture of shelf $\mathrm{D}$ is shown on the right.

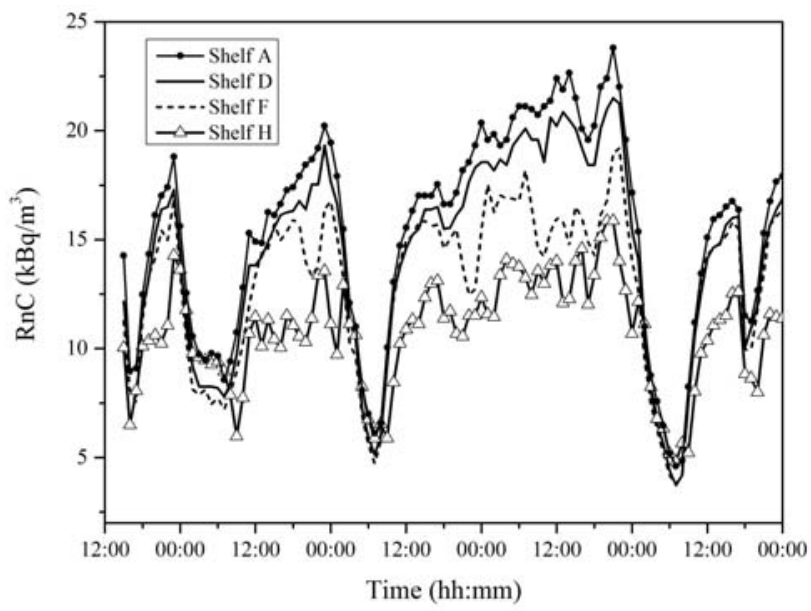

Fig. 2. Radon concentration fluctuations for different shelves; as each shelf is identified by a different $x$-axis value, this graph clearly show a gradient along the $x$-coordinate.

In the days before the exposures, the indoor radon concentration showed a regular behaviour, with daily fluctuations typical of a natural underground site: the radon concentrations decrease during night-time and increase during the day (Fig. 2). Moreover, the absence of any significant radon concentration gradient along the $y$-axis $(y: 0-80 \mathrm{~cm})$ and the $z$-axis $(z: 0-100 \mathrm{~cm}$; see Fig. 1$)$ was found, whereas a very clear gradient was observed for the $x$-axis (Fig. 2) in which the data of the monitors placed in the shelves were reported: it can be observed that the radon concentration decrease from shelf A to shelf $\mathrm{H}$.

Then radiological characterization considered gamma dose rate evaluations was executed by means of a plastic scintillator detector (Automess 6150 $\mathrm{AD}-\mathrm{b}, \mathrm{GmbH}$ ). The radiation field in the volume occupied by the shelves was calculated by interpolation of the experimental data using as weights the $1 / r^{2}$ function, with the software package $\mathrm{R}$ [7]. It turned out that the radiation field is not uniform, probably because of the complex morphology of the cavity itself. During the intercomparison exposures, the radiation field occupied by the shelves was evaluated by means of a set of thermoluminescence dosimeters (TLD-100): in this way, it was possible to assign to each set of devices a proper gamma dose rate value. Data ranged from a minimum of 0.403 and $0.471 \mu \mathrm{Gy} \cdot \mathrm{h}^{-1}$ to a maximum of 0.541 and $0.687 \mu \mathrm{Gy} \cdot h^{-1}$ with an average value of 0.475 and $0.611 \mu \mathrm{Gy} \cdot \mathrm{h}^{-1}$ during the lower and the higher exposures, respectively. All participants received the information about all the relevant environmental data (see Table 1), referred to the two intercomparison exercise exposures. The meteorological and environmental parameters (temperature, pressure and humidity) were gathered by an Automatic Weather Station MAWS201 (Vaisala, Finland).

\section{Logistic arrangements and exposure}

The participants sent two sets of dosimeters (10 radon devices each): one test at low radon exposure (around $600 \mathrm{kBq} \cdot \mathrm{h} \cdot \mathrm{m}^{-3}$ ) and one at high- 
Table 1. Environmental data sent to all the participants. The data related to temperature, pressure and humidity are the average values obtained during the measurement period (uncertainty reported with $k=1$ )

\begin{tabular}{lcc}
\hline & Low exposure & High exposure \\
\hline Height (above sea level) of the exposure site & $720 \mathrm{~m}$ a.s.l. \\
Height (above sea level) of the storing place of the dosimeters & $253 \mathrm{~m}$ a.s.l. \\
$\quad$ (Ivrea, Arpa Piemonte Laboratory) & $0.150 \pm 0.015$ \\
Gamma dose rate of the storing place $\left[\mu \mathrm{Gy} \cdot \mathrm{h}^{-1}\right]$ & 46 & 382 \\
Duration of exposure $[\mathrm{h}]$ & $9.3 \pm 0.2$ & $9.2 \pm 0.2$ \\
Temperature $\left[{ }^{\circ} \mathrm{C}\right]$ & $931.8 \pm 0.3$ & $930.8 \pm 0.3$ \\
Pressure $[\mathrm{hPa}]$ & $94.8 \pm 3.0$ & $94.5 \pm 3.0$ \\
Humidity $[\% \mathrm{RH}]$ & & \\
\hline
\end{tabular}

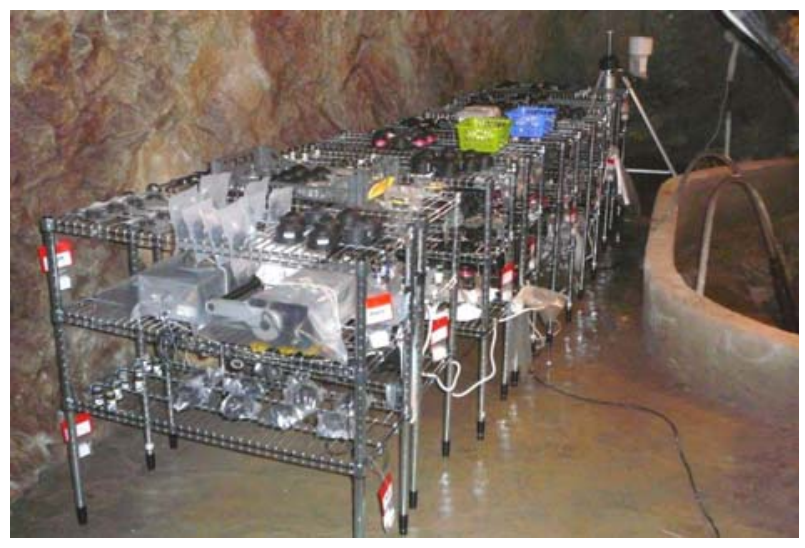

Fig. 3. The dosimeters on the shelves during the exposure.

-exposure value (about $8000 \mathrm{kBq} \cdot \mathrm{h} \cdot \mathrm{m}^{-3}$ ). All the dosimeters were previously stored at ARPA Laboratory (Ivrea) in radon proof bags, until the day before the beginning of the exercises. Radon proof bags were opened directly in Lurisia in open air only for the time needed for the arrangement of the dosimeters and the separation of transits (two dosimeters for each group), then sealed again. The positioning of the dosimeters on the shelves inside the tunnel required no more than $10 \mathrm{~min}$ (Fig. 3). The two tests were performed in sequence: the first was the higher one. During the exposures, the indoor radon concentration was controlled by the reference monitors. At the end of the tests, all exposed dosimeters and transits ones were collected and stored in an

Table 2. Reference values for the low-exposure test as a function of the position ( $x$-axis value). There was no statistically significant variation of radon concentration along the $x$-axis

\begin{tabular}{lcc}
\hline Shelf & $\begin{array}{c}X \\
{[\mathrm{~cm}]}\end{array}$ & $\begin{array}{c}\text { Radon reference value } \\
{\left[\mathrm{kBq} \cdot \mathrm{h} \cdot \mathrm{m}^{-3}\right]}\end{array}$ \\
\hline $\mathrm{A}$ & 0 & $616 \pm 31$ \\
$\mathrm{D}$ & 173 & $595 \pm 30$ \\
$\mathrm{H}$ & 369.5 & $613 \pm 31$ \\
\hline
\end{tabular}

Table 3. Reference values for the high-exposure test as a function of the position ( $x$-axis value). A significant variation of radon concentration along the $x$-axis was observed

\begin{tabular}{lcc}
\hline Shelf & $\begin{array}{c}X \\
{[\mathrm{~cm}]}\end{array}$ & $\begin{array}{c}\text { Radon reference value } \\
{\left[\mathrm{kBq} \cdot \mathrm{h} \cdot \mathrm{m}^{-3}\right]}\end{array}$ \\
\hline $\mathrm{A}$ & 0 & $8712 \pm 348$ \\
$\mathrm{D}$ & 173 & $8167 \pm 327$ \\
$\mathrm{H}$ & 369.5 & $6029 \pm 241$ \\
\hline
\end{tabular}

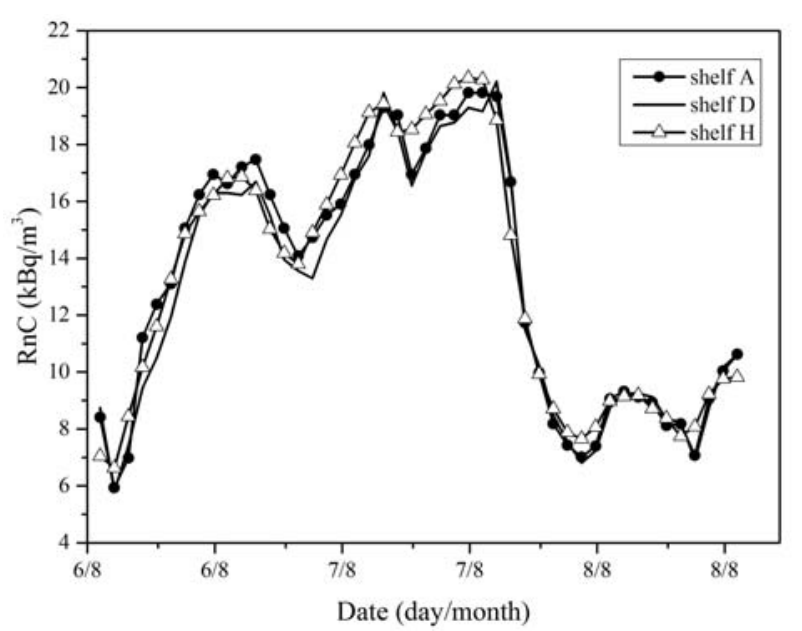

Fig. 4. Radon levels during the low-exposure test.

outdoor place in Ivrea for several days, before being sealed again in radon proof bags.

INMRI determined the radon reference values and the related uncertainties, computed with $k=1$ (see Tables 2 and 3), considering only experimental data provided by monitors calibrated at INMRI, placed on the shelves A, D and H. During the intercomparison exercise tests, two quite different situations occurred. Indeed, during the low-exposure test, no statistically significant variation along the three axis, $x, y$ and $z$, was found (Fig. 4); therefore, a unique reference value was evaluated by simply averaging the three experimental data shown in Table 2: the radon reference value of the low exposure was equal to $608 \pm 74 \mathrm{kBq} \cdot \mathrm{h} \cdot \mathrm{m}^{-3}$.

Conversely, for the high-exposure test, a significant variation of radon concentration along the $x$-axis was observed (Fig. 5). In this case, the radon reference value for each set was, therefore, evaluated by fitting the experimental data (Table 3) with a second-degree polynomial curve (Fig. 6). It was thus possible to obtain the reference radon exposure value for each exposed set on the basis of the relative $x$-coordinate. The calculated radon exposure values ranged from 5900 to $8714 \mathrm{kBq} \cdot \mathrm{h} \cdot \mathrm{m}^{-3}$. The overall uncertainty of the radon reference value for each set was evaluated, accounting for three different contributions: the uncertainty of the reference monitor, as estimated by INMRI (4-5\%), the uncertainty because of the interpolation $(0.5-2 \%)$ and a contribution because of the uncertainties related to the exposure time and the position of the sets on the shelves $(2 \%)$. The overall uncertainty on the 


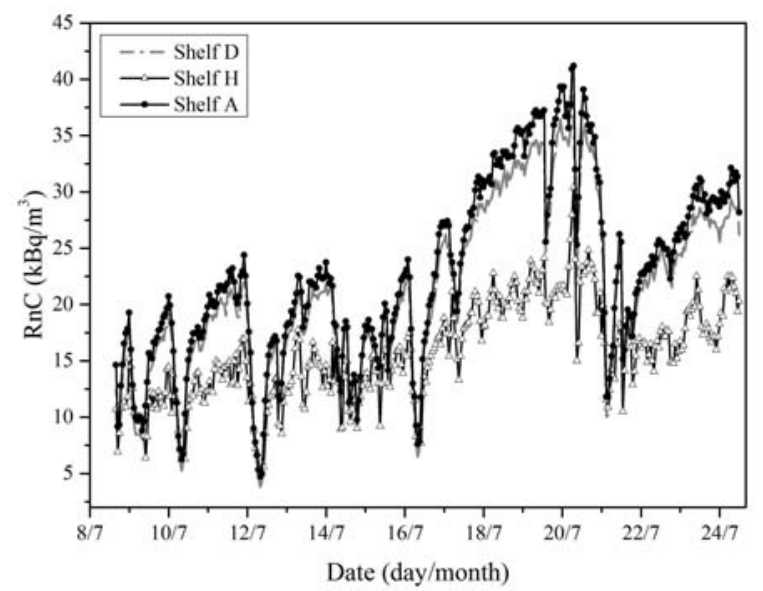

Fig. 5. Radon levels during the high-exposure test.

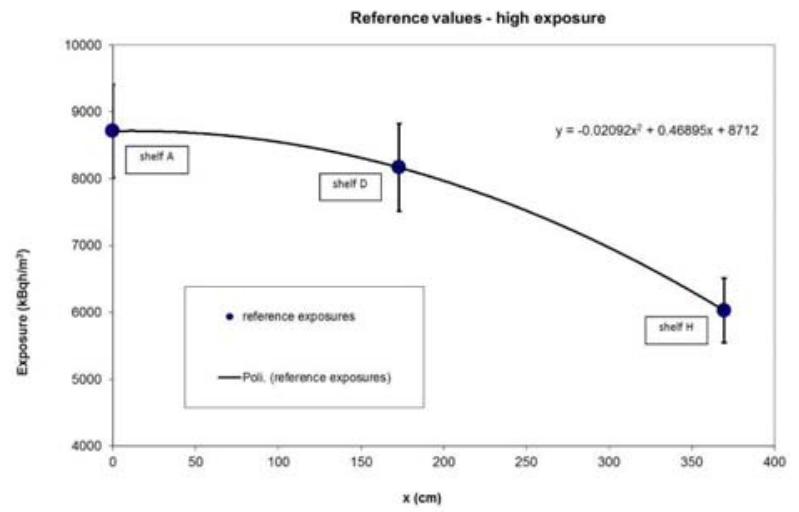

Fig. 6. High-exposure experimental reference values and the polynomial interpolation.

reference radon values was thus estimated in the range $4.5-5 \%$ for the high exposure and $6 \%$ for the low exposure.

\section{Results and discussion}

\section{Presentation of data}

The participants submitted 50 sets, identified by a number (ID): indeed four laboratories sent a double pair of sets of dosimeters. During the intercomparison exercise, about 1000 passive devices were exposed: mostly were solid-state nuclear track detectors (SSNTD) with CR39 (64\%), LR115 (10\%) or Makrofol (2\%) as plastic detector, and also electrets (24\%). Results were submitted for 48 out of the 50 sets of dosimeters. Not all the laboratories provided data related to transits. In some case, the radon exposure results were given as a net value with the transit contribution already subtracted. For all the other cases, we determined the net values before calculating the means, medians and the standard deviation. In Fig. 7, main results referred to the low radon exposure test are shown: for each devices set, the arithmetic mean and the standard deviation are presented. The solid line is the reference radon exposure (in this case $608 \mathrm{kBq} \cdot \mathrm{h} \cdot \mathrm{m}^{-3}$ ), while the dashed lines is the related uncertainty. Similarly, in Fig. 8, results of the high-exposure test are reported. In this case, as said above, reference radon values are in the

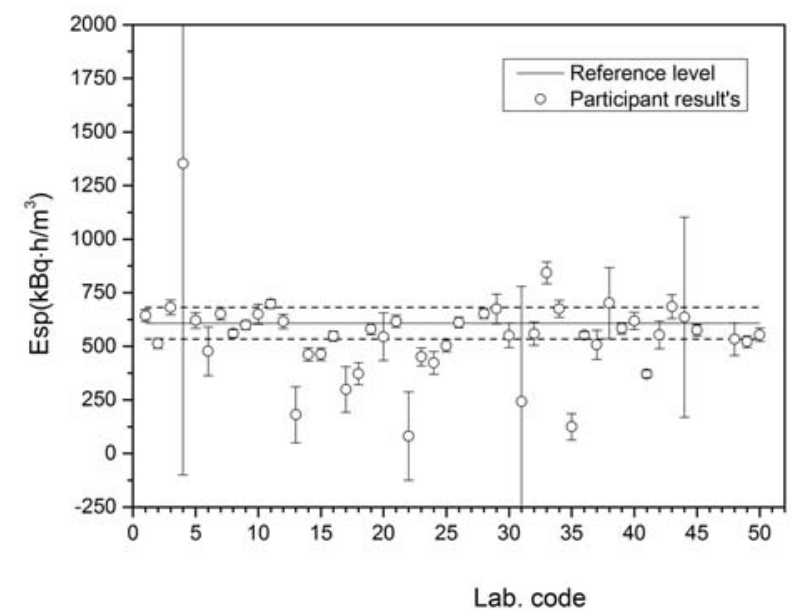

Fig. 7. Low exposure: synthesis of results of participants. For each ID, the arithmetic mean and standard deviation, respect to the reference value (grey line) and associated uncertainty ( $k=1$; grey dashed lines) are plotted.

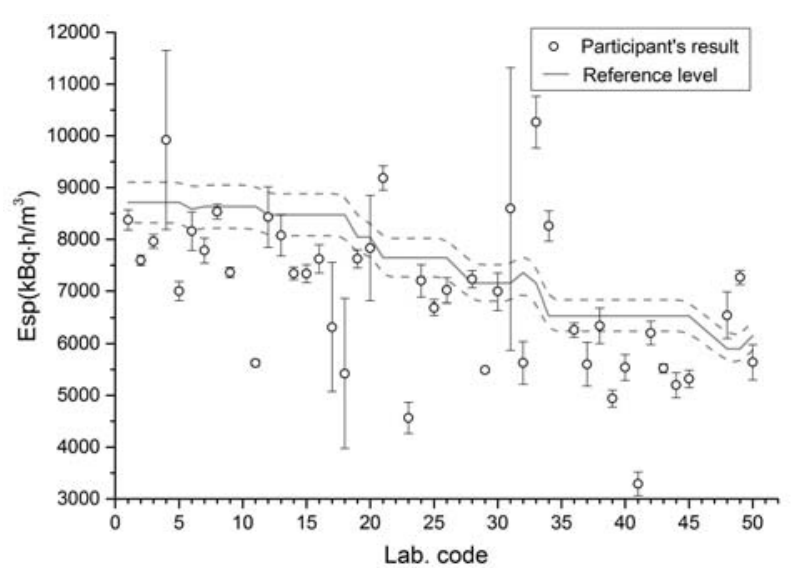

Fig. 8. High exposure: synthesis of results of participants. For each ID, the arithmetic mean and standard deviation, respect to the reference value (grey line) and associated uncertainty ( $k=1$; grey dashed lines) are plotted.

range $5900-8714 \mathrm{kBq} \cdot \mathrm{h} \cdot \mathrm{m}^{-3}$. In both tests, it can be seen that most of the participants gave results in good agreement with the reference value, even if, as a whole, a slightly underestimation trend appears.

\section{Analysis of data}

The analysis of each test results provided by participants has been done computing two indexes: the normalized error $\left(E_{n}\right)$ and the $z$-score.

$E_{n}$ is the statistical evaluation used to compare proficiency testing results where the uncertainty in the measurement result is included. The calculation of $E_{n}$ is the first evaluation used to determine the conformance or nonconformance in proficiency testing. The $E_{n}$ is given by the following equation:

$$
E_{n}=\frac{E_{i}-E_{R i}}{\sqrt{U\left(E_{i}\right)^{2}+U\left(E_{R i}\right)^{2}}}
$$

where $E_{i}$ is the arithmetic mean of the laboratory results in terms of radon exposure, $E_{R i}$ is the reference values and $U\left(E_{i}\right)$ and $U\left(E_{R i}\right)$ are the related 


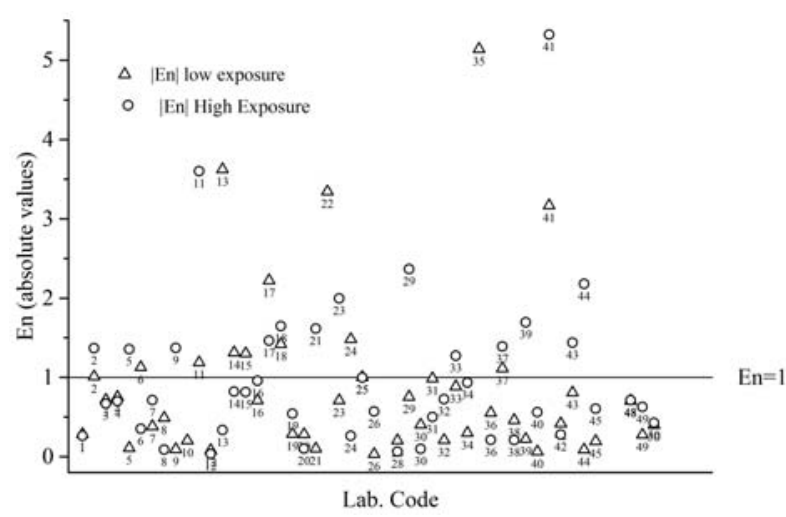

Fig. 9. Absolute value of $E_{n}$ for the low and high exposure. uncertainties. Results are satisfactory if the value of $E_{n}$ is between -1 and +1 .

The $z$-score represents a standardized measure of performance, calculated using the laboratory results. The formula to calculate standard $z$-score is given below:

$$
z_{i}=\left(E_{i}-E_{R i}\right) / \sigma_{R i}
$$

where $\sigma_{R i}$ is the standard deviation for proficiency assessment chosen as a target for the intercomparison exercise. In this exercise was decided a target of $\sigma_{R i}$ equal to $20 \%$ of the reference value. Following the indication of the ISO/IEC 17043:2010 [8] and ISO 13528:2005 [9], the laboratories with $z$-score greater than 2 (as absolute value) have been highlighted, as their values cannot be considered fully acceptable. More precisely, a $z$-score in the range 2-3 shows that some measurement problems have to be solved, while a value greater than 3 is considered unacceptable. The large majority of laboratories $(87 \%)$ showed a $z$-score lesser than 2.

In Fig. 9, $E_{n}$ values for the low and high exposures are shown, while in Fig. 10, absolute values of the $z$-score for both low and high exposures are reported. It can be observed that the criterion $E_{n}<1$ is by far more strict than the one based on the $z$-score value. In fact, in this last case, the laboratories that gave unacceptable results are much more: $28 \%$ for the low exposure and $30 \%$ for the high exposure.

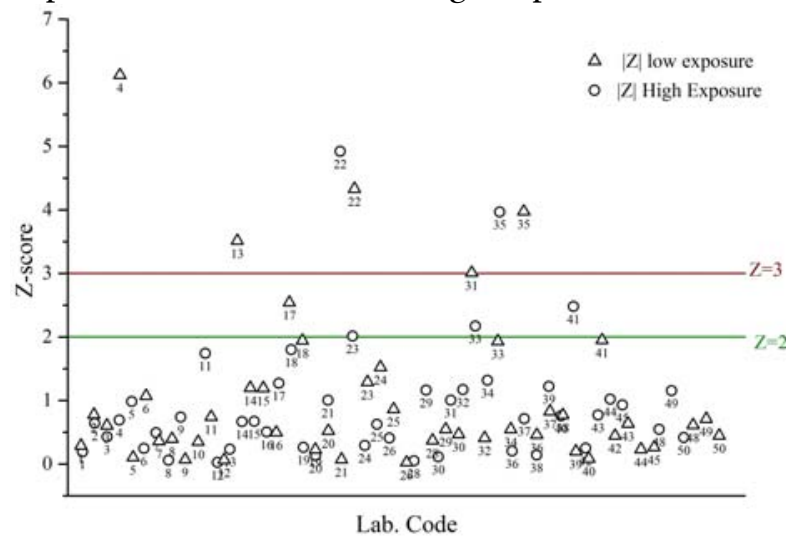

Fig. 10. Absolute values of the $z$-score for the low and high exposure. Values above 2 and 3 are highlighted: following the ISO standards, values lesser than 2 are considered acceptable. Values in the range $2-3$ give a warning. Values above 3 are considered unacceptable.

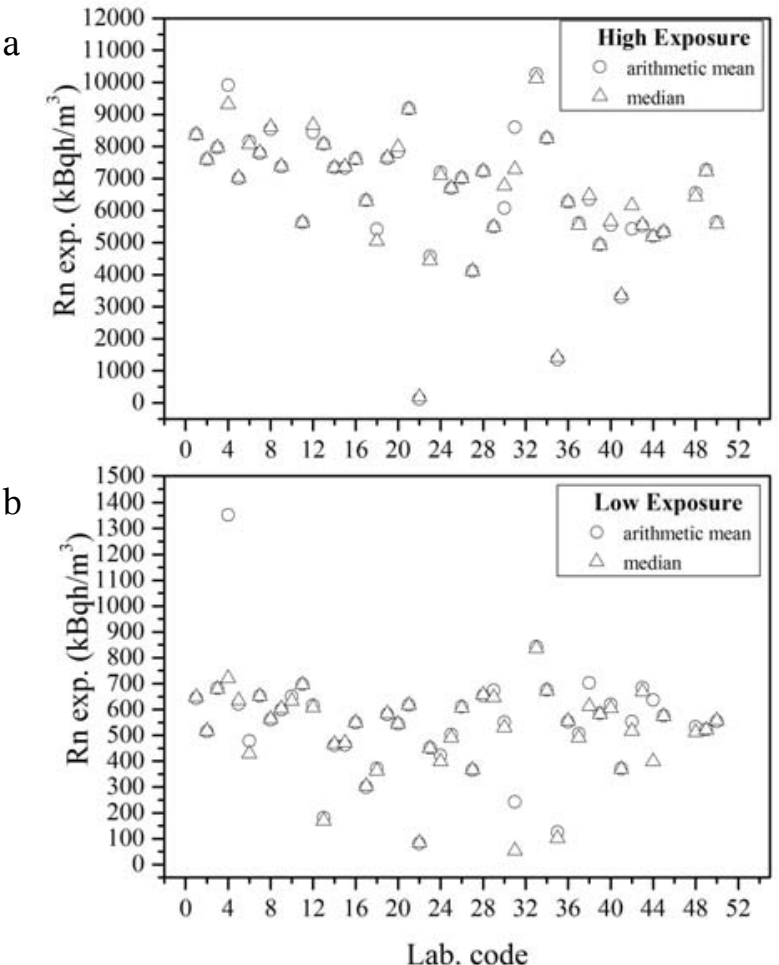

Fig. 11. Arithmetic mean vs. median. (a) High exposure; (b) low exposure.

Looking at the data provided by each laboratory, we can analyse the distribution of data related to each dosimeter for each laboratory. In particular, we can compare the arithmetic mean and the median for low and high exposure for each laboratory. In most cases, arithmetic means and medians of the net exposures were very close, showing thus a roughly symmetric distribution of data related to each set of dosimeters (Fig. 11). For the low and high exposures, respectively, only $9 \%$ and $17 \%$ of laboratories have a difference between mean and median value more than $10 \%$.

Moreover, to evaluate the performance of the laboratories taking into account results achieved in both the tests ( $E_{n}$ and the $z$-score), it is possible to use the Youden plot (Fig. 12). With this technique, the performance of the laboratory is measured

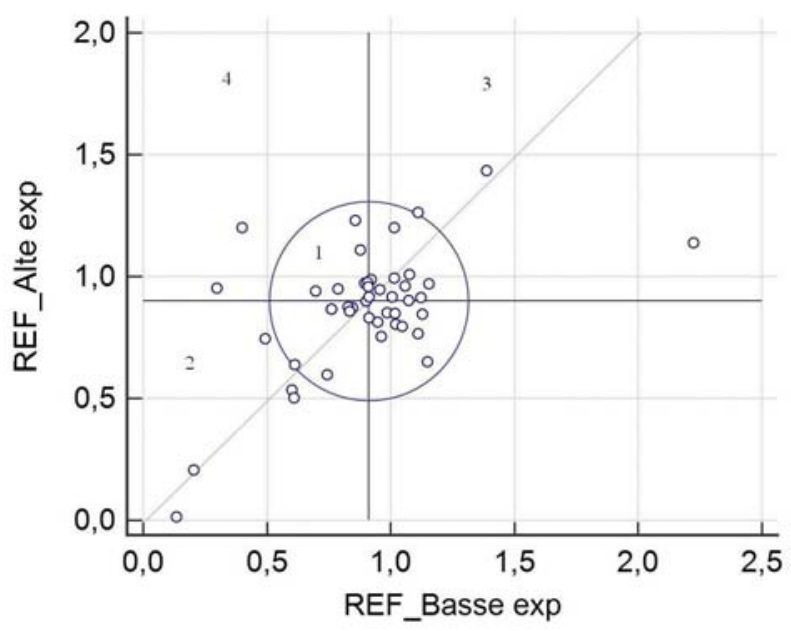

Fig. 12. Youden plot: $80 \%$ of the laboratories are within the circle representing the $95 \%$ confidence interval. 
by the distance between a point whose coordinates are given by the experimental values of the two exposures (expressed in terms of REF, which is the ratio between $E_{i}$ and the reference value $E_{R i}$ ) and the centre of a circle. In Youden plot, the distance from the bisector quantifies the relevance of systematic errors. Looking at Fig. 12, it can be seen that about $80 \%$ of the participants lies in the area 1 , limited by a circle representing the $95 \%$ confidence interval: these data can, therefore, be considered acceptable. Seven laboratories (15.5\%), whose values are outside the circle but close to the bisector (areas 2 and 3), showed a good reproducibility but poor accuracy. Only two laboratories (4.5\%) in the areas 4 and 5 gave totally unacceptable data, having, at the same time, poor accuracy and poor reproducibility.

\section{Conclusions}

In 2014, in the Marie Curie's tunnel in Lurisia, the international intercomparison of passive radon detectors under field conditions was organized. Two different tests were executed: the integrated radon exposure range for the first test was 6000-9000 $\mathrm{kBq} \cdot \mathrm{h} \cdot \mathrm{m}^{-3}$, while that for the second exercise was $600-700 \mathrm{kBq} \cdot \mathrm{h} \cdot \mathrm{m}^{-3}$.

It was thus decided not to establish a unique criterion for the evaluation of the performances. Many reasons suggested this approach: the most important one is the fact that the great difficulty and complexity typical of the in-field intercomparison would have made questionable any definition of criteria for the listing of a rank. The organizers preferred to simply calculate and discuss a number of different indicators such as normalized error $\left(E_{n}\right), z$-score and the Youden plot, without giving any ranking criteria. In this paper, comments and considerations on the results obtained by the laboratories generally followed the ISO [9] suggestions are given. The only one arbitrary choice was to assume for the parameter $\sigma_{R i}$ that appears in the $z$-score definition, a value corresponding to the $20 \%$ of the reference value.

By analysing the distribution of the results, some comment can be done: the frequency distribution of the results was asymmetrical with a prevalence of an underestimation respect to an overestimation $(28 \%$ vs. $6 \%$ of laboratories); about $66 \%$ of laboratories provide a result centred on the reference value for the low exposure; for the high exposure, an analogous trend was observed (37\% of the laboratory underestimated the results, while the $11 \%$ overestimated it).
The radon measurements services and laboratories participating to the intercomparison appreciated the possibility to test their systems during in situ exercises, with exposures having place in real conditions, which are less controlled and much more variable, very similar to the ones in which devices are normally exposed.

\section{References}

1. Daraktchieva, Z., Howarth, C. B., \& Aigar, R. (2013). Results of the 2012 HPA intercomparison of passive radon detectors. Chilton, Didcot: P.H.E. (PHE-CRCE-001).

2. Foerster, E., Beck, T., Buchröder, H., Döring, J., \& Schmidt, V. (2014). Instruments to measure radon-222 activity concentration or exposure to radon-222: Intercomparison 2014. Germany: BfS.

3. Janik, M., \& Yonehara, H. (2015). The most recent international intercomparisons of radon and thoron monitors with the NIRS radon and thoron chambers. Radiat. Prot. Dosim., 164(4), 595-600.

4. International Electrotechnical Commission. (2009). IEC 61577-4:2009 Radiation protection instrumentation - Radon and radon decay product measuring instruments - Part 4: Equipment for the production of reference atmospheres containing radon isotopes and their decay products (STAR). Geneva: IEC.

5. Gutiérrez-Villanueva, J. L., Sainz Fernandez, C., Fuente Marino, I., Quindos Lopez, L., Quindos Lopez, J., Fernandez Villar, A., Casal Ordas, S. E., Lopez Abascal, D., Artheche Laso, D., Fernandez Lopez, E., \& Quindos Poncela, L. S. (2012). International intercomparison exercise on natural radiation measurements under field conditions. PUbliCan Edicione Universidad de Cantabria.

6. Cardellini, F., Chiaberto, E., Garlati, L., Giuffrida, D., Leonardi, F., Magnoni, M., Minchillo, G., Prandstatter, A., Serena, A., Trevisi, R., Tripodi, R., \& Veschetti, M. (2016). Metrological aspects of international intercomparison of passive radon detectors under field conditions in Marie Curie's tunnel in Lurisia. Nukleonika, 61(3), 257-261.

7. R Core Team. (2016). R: A language and environment for statistical computing [computer software]. http:// www.R-project.org/

8. International Organization for Standardization. (2010). Conformity assessment - General requirements for proficiency testing. ISO/IEC 17043:2010.

9. International Organization for Standardization. (2015). Statistical methods for use in proficiency testing by interlaboratory comparison. ISO 13528:2015. 